\title{
Strategies to mitigate the risk from COVID-19 in ophthalmology
}

\author{
Ali Poostchi', Joanna K. Dereń
}

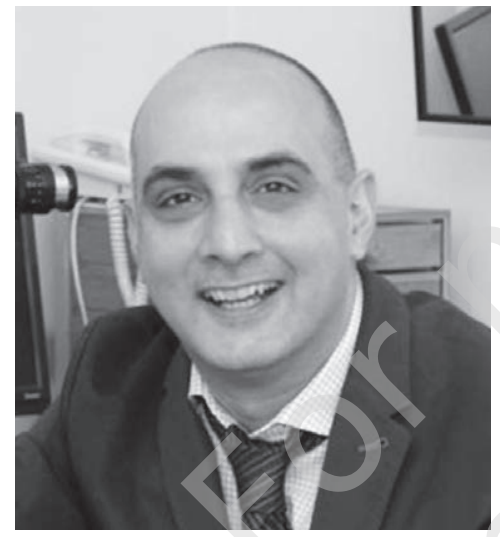

'Glaucoma Fellow, Glaucoma Service, Moorfields Eye Hospital, London, UK Joint divisional director, Moorfields North: Jonathan Clarke, MD

${ }^{2}$ Military Institute of Aviation Medicine in Warsaw, Poland Head of the Department: Radosław Różycki, MD, PhD

I have no idea what's awaiting me, or what will happen when this all ends. For the moment I know this: there are sick people and they need curing.

H I G H L I G H TS

Minimizing the risk of disease transmission of COVID-19 by using a combination of distancing, shielding and disinfection will help medical practitioners to safely deliver uninterrupted high quality ophthalmic care.
Albert Camus, The Plague

\begin{abstract}
Many countries, including Poland, are currently dealing with a second wave of COVID-19 infections. There is a pressing need to adapt to the new reality and develop new ways of working in order to deliver essential services safely. In the medical sector, there is a special need to deliver uninterrupted high quality care while minimizing the risk of disease transmission. In this article, we summarize the evidence on the transmission dynamics and ophthalmic features of COVID-19 and suggest a hierarchical approach to infection control, in order to help practitioners understand and mitigate the risks they face each day.
\end{abstract}

Key words: COVID-19, personal protective equipment, face mask, face shield, eye protection 


\section{INTRODUCTION}

The 2020 coronavirus pandemic has had a profound impact on society and on the delivery of healthcare. While Poland was relatively spared during the first wave of infections, a cluster of cases around a Silesian mine and ongoing community transmission highlighted the need for continued vigilance. The impact of the second waves continues to be felt and as lockdowns ease and work is restarted there remain challenges around balancing infection control measures with efficient throughput of patients to address the backlog and mitigate the effects of delays in treatment. The aim of this article is to provide practical guidance on simple but effective infection control strategies that can be used by ophthalmologists in their daily practice.

\section{TRANSMISSION DYNAMICS}

Transmission of SARS-CoV-2, the virus responsible for COVID-19 is thought to occur via infected droplets which originate from the respiratory tract of infected individuals.

These are traditionally divided in to large droplets and small droplet nuclei [1]. Larger droplets are affected by gravity and settle on surfaces up to $2 \mathrm{~m}$ away, creating fomites. Infection then occurs either through direct contact or after touching a contaminated surface. Masks are thought to be more effective at protecting others from transmission rather than the wearer because they capture larger droplets as they leave the nose and mouth and before they can evaporate into smaller droplet nuclei which are harder to capture.

Smaller droplet nuclei $(\leq 5-10 \mu \mathrm{m})$ evaporate before reaching the ground leaving aerosolised residues that remains in air for longer periods and travel further. They can also be inhaled further down into the respiratory tract than droplets.

These three modes of transmission are commonly described as droplet, fomite and airborne (or aerosol) transmission respectively.

The relative importance of each mode and the risk of viral shedding from asymptomatic individuals is not fully known and will vary according to the viral strain. Early reports from WHO suggested that most spread was via droplets and fomites [2] but aerosols containing viable virus have been produced in experimental conditions [3]. The initial reproduction number (R0) of SARS-CoV-2 is thought to be 2-3 which is more consistent with droplet and fomite rather than airborne transmission [4]. By contrast, measles which is known to spread via all three routes and has an R0 of $10-18$.

\section{PHYSICAL DISTANCING}

The apparent effectiveness of physical distancing and facemasks also supports fomites and droplets as the main vectors of transmission. A recent WHO funded systematic review of infection control measures found that the risk of transmission was five times lower at distances beyond one meter and that protective benefit doubled every meter the distance was increased beyond this [5].

\section{MASKS}

Masks are normally classified according to filtration efficiency. Within the European Union, filtering face piece (FFP) 1, 2 and 3 will filter 80\%, 94\% and 99\% of airborne particles, respectively. In the United States, minimum filtration efficiency is included within the grading system, so that FFP3 masks are equivalent to N99, while FFP2 are similar to N95 masks. Surgical face masks are not sealed against the face and allow transmission of particles around the edges but are effective as 'source control' and have been shown to reduce the risk contamination during cataract surgery [6].

While higher grade masks offer greater protection, this depends on proper use and in clinical settings, levels of adherence vary. Touching the inside or outside of masks when removing or adjusting them allows transmission via fomite spread and largely negates their value. Studies on influenza have shown a greater benefit from the use of N95 respirators in controlled experimental settings [7] but not in day to day practice in outpatient clinics. A large pragmatic randomised control trial showed no benefit from using N95 respirators compared to surgical facemasks in reducing respiratory infections among health care workers [8]. This was supported by a subsequent meta-analyses which found the benefits higher filtration respirators were likely offset by discomfort with prolonged use leading more frequent manipulation or reduced compliance [9].

In the WHO review, there was some observational evidence that N95 masks were more effective than surgical facemasks at reducing COVID-19 transmission, but this could be have confounded by the use of other strategies in units where N95 masks were available.

\section{OTHER SHIELDS}

Face mask also make communication more difficult through muffling and by preventing lip reading. The use face shields and other clear barriers can be helpful to overcome this, while also providing some eye protection. Shields are easier to manufacture and can be more easily decontaminated and reused [4] but do have gaps around the edges and so are commonly used in combination with facemasks rather than alone. In one study, the addition of 
face shields to masks and gloves eliminated infections in a group community health workers assigned to trace family contacts of patients with COVID-19 [10]. When a higher level of protection is required, low cost clear ventilated hood/powered air purifying respirators (PAPRs) can be used and these have been successfully trialled with a view to widespread adoption [11].

Barrier shielding attached to equipment also appears worthwhile.One study using a laser particle counter found that face masks and enlarged perspex breath shields had similar efficacy in reducing airborne and droplet transmission across a slit lamp [12]. Other studies set in ophthalmic outpatients and theatres have attempted to directly visualise sprayed dye droplets but these experiments only show the distribution of larger droplets as aerosols are too small to be seen by the naked eye.

\section{EYE PROTECTION}

The use of eye protection to prevent infection via the conjunctiva has been shown to be important against SARS and MERS. In the WHO review it was thought likely to confer additional benefits [5] though there was no specific data for COVID-19. Wearing glasses for extended periods may also be helpful and one epidemiological study found that very few patients who had been hospitalised with COVID-19 wore glasses (6\%) compared to the local population (32\%) [13].
There is some ongoing uncertainty regarding the risk of transmitting infection from the eye. SARS-CoV-2 RNA has been found to present in tears but the overall risk of transmission is thought to be low [14].

\section{RISK FROM CLINICAL PROCEDURES}

Unlike adenovirus, SARS-CoV-2 is an enveloped virus and tonometer heads can be quickly decontaminated using alcohol or $0,5 \%$ sodium hypochlorite. In the operating room, povidone iodine is routinely applied preoperatively and has been shown to be effective against SARS-CoV-1 [15].

While some aerosolisation can occur during cataract surgery, the aqueous is initially replaced with viscoelastic prior to phacoemulsification and any particles that remain present will be heavily diluted by the continual circulation of BSS.

The risk from coughing or sneezing from an infected patient during a procedure remains but can be mitigated through adequate draping, decontamination between patients and pre-operative testing and self isolation of patients starting 2 weeks prior to surgery.

\section{HIERARCHY OF CONTROL}

When developing systems to mitigate and manage risk, the concept of the hierarchy of control adapted from occupational safety research can be helpful (tab. 1).

\begin{tabular}{|c|c|c|}
\hline Effectiveness & Hazard control & Example \\
\hline \multirow{3}{*}{5 (most effective) } & \multirow{3}{*}{ Elimination } & minimise clinic visits, use phone consultations or telemedicine where possible \\
\hline & & isolate and test patients prior to surgery \\
\hline & & consider bilateral simultaneous cataract surgery where felt safe to do so \\
\hline 4 & Substitution & not applicable \\
\hline \multirow{3}{*}{3} & \multirow{3}{*}{ Engineering control } & good ventilation, open windows and allow air to circulate \\
\hline & & perspex shielding on slit lamps and in front of reception areas \\
\hline & & adequate draping during surgery \\
\hline \multirow{5}{*}{2} & \multirow{5}{*}{$\begin{array}{l}\text { Administrative } \\
\text { controls }\end{array}$} & encourage regular hand washing and cleaning \\
\hline & & disinfect slit lamp between patients with soap or alcohol \\
\hline & & consider introducing a one-way system to reduce contact between patients \\
\hline & & ensure adequate spacing in waiting areas and avoid overcrowding \\
\hline & & avoid close contract except for when inevitable during examination \\
\hline 1 (least effective) & $\begin{array}{c}\text { Personal protective } \\
\text { equipment }\end{array}$ & masks/glasses/face shields \\
\hline
\end{tabular}


Where possible, the hazard should be removed or replaced and this can be achieved by minimising unnecessary appointments and allowing home working for administrative tasks. The introduction of simultaneous bilateral cataract surgery has been suggested as one measure that could be used to reduce the number of visits but this should be balanced against the risk of bilateral postoperative endophthalmitis [16].

Further down the hierarchy, the use of engineering controls, such as ventilation systems and physical barriers, and administrative controls including education around physical distancing and frequent hand washing provide further protection.

Finally, when direct contact with individuals at risk is unavoidable, the meticulous use of personal protective equipment (PPE) including face masks and eye protection or face shields coupled with good situational awareness and thorough decontamination, will minimise the risk of transmission and allow safe working (tab. 2).

\section{TABLE $\quad 2$}

\section{Effectiveness of different control methods on risk of viral transmission (adapted from Chu et al. [5]).}

\begin{tabular}{|c|c|c|c|}
\hline Method & Control group & Intervention group & Adjusted OR* \\
\hline Physical distancing & short distance < 1 m (13\%) & greater distance > 1 m (3\%) & $5.6(2.6-11.1)$ \\
\hline Face mask & no face mask (17\%) & face mask worn (3\%) & $6.7(2.9-14.3)$ \\
\hline Eye protection & no eye protection (16\%) & eye protection worn (6\%) & $4.5(2.6-8.3)$ \\
\hline
\end{tabular}

The risk of transmission in different circumstances is shown in brackets.

*Adjusted odds ratio with $95 \%$ confidence intervals.

\section{CORRESPONDENCE}

Ali Poostchi, MD

Glaucoma Fellow, Moorfields Eye Hospital, London, UK

162 City Rd, London EC1V 2PD

e-mail: a.poostchi@nhs.net

\section{ORCID}

Ali Poostchi - ID - http://orcid.org/0000-0002-1014-2016

\section{References}

1. Wells WF. On air-borne infection. Study II. Droplets and Droplet Nuclei. Am J Epidemiol. 1934; 20: 611-8.

2. World Health Organization. Report of the WHO-China Joint Mission on Coronavirus Disease 2019 (COVID-19). 2020.

3. van Doremalen N, Bushmaker T, Morris DH et al. Aerosol and Surface Stability of SARS-CoV-2 as Compared with SARS-CoV-1. N Engl J Med. 2020: NEJMc2004973.

4. Perencevich EN, Diekema DJ, Edmond MB. Moving Personal Protective Equipment Into the Community: Face Shields and Containment of COVID-19. JAMA. 2020; 323: 2252-3.

5. Chu DK, AkI EA, Duda S et al. Physical distancing, face masks, and eye protection to prevent person-to-person transmission of SARS-CoV-2 and COVID-19: a systematic review and meta-analysis. Lancet. 2020; 395: 1973-87.

6. Alwitry A, Jackson $\mathrm{E}$, Chen $\mathrm{H}$ et al. The use of surgical facemasks during cataract surgery: is it necessary? Br J Ophthalmol. 2002; 86: 975-7.

7. Noti JD, Lindsley WG, Blachere FM et al. Detection of Infectious Influenza Virus in Cough Aerosols Generated in a Simulated Patient Examination Room. Clin Infect Dis. 2012; 54: 1569-77.

8. Radonovich LJ, Simberkoff MS, Bessesen MT et al. N95 Respirators vs Medical Masks for Preventing Influenza Among Health Care Personnel: A Randomized Clinical Trial. JAMA. 2019; 322: 824-33.

9. Long Y, Hu T, Liu L et al. Effectiveness of N95 respirators versus surgical masks against influenza: A systematic review and meta-analysis. J Evid Based Med. 2020: jebm.12381.

10. Bhaskar ME, Arun S. SARS-CoV-2 Infection Among Community Health Workers in India Before and After Use of Face Shields. JAMA. 2020; 324: 1348. 
11. Elkington $P$, Morgan H. Personal respirators for population level control of the COVID19 pandemic. J Infect. 2020; 81: 318-56.

12. Poostchi A, Kuet M-L, Pegg K et al. Efficacy of slit lamp breath shields. Eye (Lond). 2020; 34: 1185-6.

13. Zeng W, Wang X, Li J et al. Association of Daily Wear of Eyeglasses With Susceptibility to Coronavirus Disease 2019 Infection. JAMA Ophthalmol. 2020; 138: 1196.

14. Seah IYJ, Anderson DE, Kang AEZ et al. Assessing Viral Shedding and Infectivity of Tears in Coronavirus Disease 2019 (COVID-19) Patients. Ophthalmology. 2020; 127(7): 977-9. Available at: https://www.aaojournal.org/article/S0161-6420(20)30311-0/abstract (access: 24.04.2020).

15. Kariwa H, Fujii N, Takashima I. Inactivation of SARS coronavirus by means of povidone-iodine, physical conditions and chemical reagents. Dermatology (Basel). 2006; 212(suppl 1): 119-23.

16. Li O, Kapetanakis V, Claoué C. Simultaneous Bilateral Endophthalmitis after Immediate Sequential Bilateral Cataract Surgery: What's the Risk of Functional Blindness? Am J Ophthalmol. 2014; 157: 749-51.e1.

Authors' contributions:

Ali Poostchi: 60\%; Joanna K. Dereń: 40\%.

Joanna K. Dereń conceived the paper, developed the outline and prepared the ma-

nuscript for publication. Ali Poostchi wrote the first draft. Both authors contributed to

the literature search, critically revised the manuscript and approved the final paper.

Conflict of interest:

None.

Financial support:

None.

Ethics:

The content presented in the article complies with the principles of the Helsinki

Declaration, EU directives and harmonized requirements for biomedical journals. 